\title{
ANTI-ANGIOGENESIS EN LA FISIOPATOLOGÍA DE LA PREECLAMPSIA. ¿LA PIEDRA ANGULAR?
}

\author{
Rommel Omar Lacunza Paredes ${ }^{1,3}$, Jorge Avalos Gómez ${ }^{2,3}$
}

\begin{abstract}
RESUMEN
La preeclampsia es la enfermedad más enigmática de la medicina fetal, recientes descubrimientos nos acercan a poder completar la compleja cascada fisiopatológica de la disfunción endotelial materna en preeclampsia. Los factores angiogénicos y el desequilibrio producido por el aumento de la producción placentaria de factores antiangiogénicos se constituyen como una sólida teoría para unificar la disfunción endotelial materna y la hipoperfusión placentaria.
\end{abstract}

Palabras clave: Preeclampsia; Disfunción endotelial; Disbalance angiogenico (Fuente: DeCS BIREME)

\section{ANTI-ANGIOGENESIS IN THE PHYSIOPATHOLOGY OF PREECLAMPSIA. THE ANGULAR STONE?}

\begin{abstract}
Preeclampsia is the most enigmatic disease of fetal medicine, recent discoveries bring us closer to being able to complete the complex physiopathological cascade of maternal endothelial dysfunction in preeclampsia. The angiogenic factors and the imbalance produced by the increased placental production of anti-angiogenic factors constitute a solid theory to unify maternal endothelial dysfunction and placental hypoperfusion.
\end{abstract}

Keywords: preeclampsia, Endothelial dysfunction, angiogenic imbalance(Source: MeSH NLM).

\section{INTRODUCCIÓN}

La historia. En la medicina materno fetal la preeclampsia es una de las enfermedades con mayor impacto en la salud pública; sin embargo, los múltiples vacíos e interrogantes en los eventos fisiopatológicos que explican el cuadro clínico materno redundan en grandes limitaciones en su manejo. El mejor reflejo de esta realidad es que actualmente el único tratamiento realmente efectivo es el término de la gestación.

La preeclampsia es la primera causa de morbilidad (ingresos a $\mathrm{UCl}$, estancias hospitalarias prolongadas, politransfusion de hemoderivados, múltiples cirugías, prematuridad iatrogénica, etc) y mortalidad materna en las principales ciudades de nuestro país. El entendimiento de sus mecanismos nos ayudará a mejorar nuestro manejo y en el futuro establecer un tratamiento que frene la cascada fisiopatológica, pudiendo evitar así el término iatrogénico de la gestación'.

El paulatino descubrimiento de la fisiopatología materna que origina la preeclampsia ha ido cambiando nuestra manera de entender la enfermedad a través de la historia. El descubrimiento de la medición de la presión arterial por Nikolay Korotkov estableció un hito, al poder diferenciar la presión diastólica de la sistólica haciendo factible el uso del biomarcador más valioso hasta la actualidad. En conjunto la unión de hipertensión arterial, proteínas en orina y edemas dan origen a la aparición del término preeclampsia para describir una serie de eventos que antecedían a la eclampsia ${ }^{2}$. A inicios del siglo veinte se describe el estado prodrómico de la toxemia gravídica (eclampsia) caracterizado por cefalea, fotopsias, acufenos y epigastralgia denominándolos "signos premonitorios", actualmente sabemos que estos signos son ya una manifestación cerebral producida por alteraciones en la barrera hematoencefalica ${ }^{3,4}$. Los modelos fisiopatológicos hasta el momento solo han resultado en la comprensión parcial de los fenómenos moleculares que ocasionan la preeclampsia, por ello las estrategias de predicción y prevención han sido fallidas.

\section{Fisiopatología: ¿Por dónde empezar?}

La preeclampsia es la enfermedad de las teorías, por lo que al abordar el estudio de la fisiopatología muchas veces se confunden conceptos haciendo engorrosa la comprensión de los eventos moleculares. Por esta razón se ha propuesto dividir esta enfermedad en dos estadios: asintomático y sintomático (figura 1), para una mejor comprensión de los eventos fisiopatológicos de la enfermedad más compleja en el ámbito de la medicina materno fetal

\footnotetext{
Médico Gineco-Obstetra. Hospital Nacional Daniel Alcides Carrión, Callao-Perú. Centro de Medicina Fetal. CENMEF, Lima-Perú

2 Médico Gineco-Obstetra. Hospital Nacional Dos de Mayo, Lima-Perú.

3 Fetalis, Grupo de Medicina Materno Fetal

Citar como: Lacunza RO, Avalos J. Anti-angiogenesis en la fisiopatología de la preeclampsia. ¿la piedra angular?. Rev Peru Investig Matern Perinat. 2019;8(4): 48-53

DOI https://doi.org/10.33421/inmp.2019173
} 


\section{Figura 1. Estadios fisiopatológicos de la preeclampsia.}

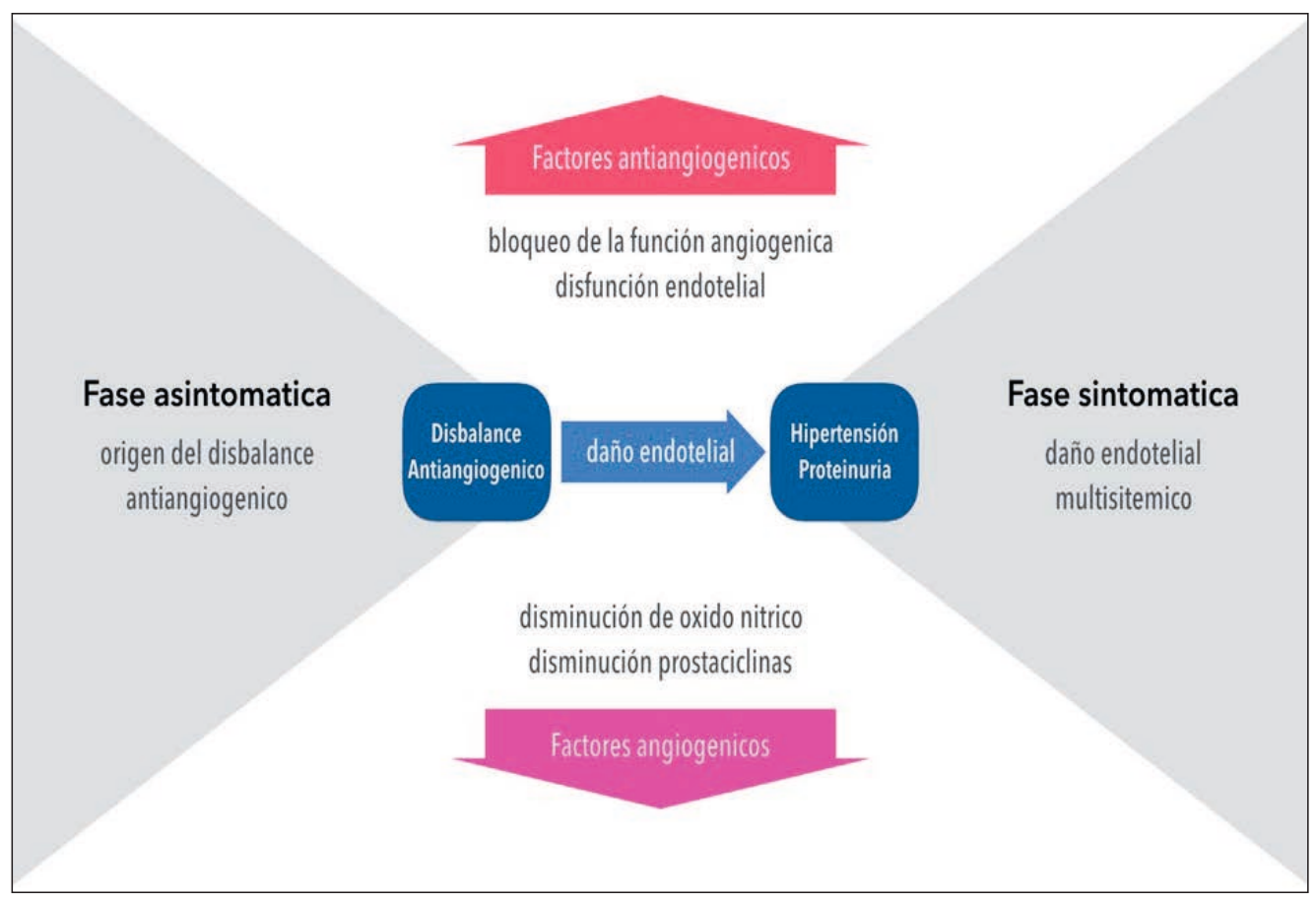

\section{Estadio Sintomático: iniciando por el final}

Como la mayoría de entidades patológicas del ser humano, la preeclampsia manifiesta sus signos y síntomas cuando ya existe un daño patente en el órgano diana, lo que significa que la noxa que desencadena la cascada fisiopatológica actúa desde hace mucho, pero tardíamente pone en evidencia disfunción del órgano para nuestra evaluación clínica. La literatura demuestra que el órgano diana en la preeclampsia es el endotelio materno, y son las manifestaciones clínicas en distintos órganos producidas por daño funcional y estructural del endotelio vascular (disfunción endotelial) ) $^{5-7}$.

Hasta la actualidad los estudios siguen intentando demostrar la sustancia "toxica" responsable del síndrome materno al cual denominamos preeclampsia. Se han investigado innumerables moléculas dependiendo de la postura teórica del investigador: inmunología, genética, vascular, infecciosa, fetal, placentaria, etc. Una de las primeras muestras de ello fue el genial experimento de $\mathrm{Page}^{8}$ en 1938 , en el cual transfundió $100 \mathrm{ml}$ de sangre de una puérpera con eclampsia a una gestante del tercer trimestre; provocando en ella cianosis, consolidación pulmonar, taquicardia, escalofrió e incremento de presión arterial; síntomas que se resolvieron espontáneamente a las 36 horas. Estos hallazgos no se lograron replicar cuando la transfusión se realizó a pacientes puérperas o post aborto. Basado en estas observaciones Page sugiere que las gestantes son vulnerables a esta "sustancia" y es necesaria la presencia de un órgano precursor (placenta) para que el estado hipertensivo persista. Actualmente a la luz de la evidencia se propugna al desequilibro angiogénico como la "sustancia presora" responsable de la "toxemia" de origen placentario (preeclampsia).

Revisaremos brevemente la función de los factores angiogénicos en el endotelio, para poder entender porque el desequilibrio nos lleva a la disfunción endotelial. En la gestación normal los mecanismos adaptativos de la madre establecen un estado de equilibrio entre la angiogenesis y antiangiogenesis ${ }^{7}$. El disbalance antiangiogénico se caracteriza por incremento de factores antiangiogénicos, entre los que se encuentra principalmente la forma soluble del receptor 1 del factor de crecimiento endotelial vascular (sFlt-1 por sus siglas en ingles fms-like-tyrosine-kinase receptor) y la forma soluble de la endoglina (sEng). Así también, la reducción de los factores angiogénicos como: factor de crecimiento endotelial vascular (VEGF por sus siglas en ingles vascular endotheial growth factor) y el factor de crecimiento placentario (PIGF por sus siglas en ingles placenta growth factor).

El endotelio vascular normal mantiene el control del tono del musculo liso de la pared vascular, regulando la liberación de sustancias vasoconstrictoras y vasodilatadoras (principalmente a través de la producción de óxido nítrico (ON) y prostaciclinas); así también, la función de anticoagulación, anti-agregante plaquetaria y fibrinolitica son mantenidas mediante la liberación de diversos factores solubles. La disfunción endotelial es la expresión del desequilibrio en las sustancias endoteliales, lo que produce aumento de la vasoconstricción, inflamación, expresión de moléculas de adhesión (activación endotelial) y alteración en la permeabilidad vascular.

En las gestantes que desarrollan preeclampsia las múltiples moléculas producidas por la placenta (trofoblasto hipóxico) son liberadas a la circulación materna (factores angiogénicos, interleuquinas, especies reactivas de oxígeno, fragmentos de trofoblasto, entre otras) generando la disfunción endotelial materna9 ${ }^{9}$. Si bien esta revisión se centra en los factores angiogénicos, es imposible soslayar la compleja interacción con otras sustancias en la producción de la disfunción endotelial, para ello podemos recomendar al lector otras revisiones ${ }^{5-7,10}$. 
En condiciones normales los factores antiangiogenicos sFlt-1 y sEng bloquean la unión del VEGF y TGF-Beta1 a sus receptores celulares al ligarse a ellos en la circulación materna, impidiendo la interacción con sus receptores endógenos celulares; estableciendo un equilibrio entre la función angiogenica y antiangiogenica (Figura 2) ${ }^{7}$. Los factores angiogénicos como el VEGF, además de su acción angiogénica cumplen funciones vasodilatadoras en el endotelio vascular incrementando el influjo de calcio intracelular, potenciando la actividad de la sintetasa de ON y de la prostaciclina I2. EI PIGF (miembro de la familia de VEFG) altamente expresado en la placenta, se liga al receptor celular de VEGF-2 con menor afinidad y produce similares funciones celulares en la gestación. Así mismo, el TGF-Beta1 incrementa en el endotelio la expresión y activación de la sintetiza de ON11 (Figura 3).

Figura 2.- Mecanismo de acción de los factores angiogénicos y antiangiogenicos.

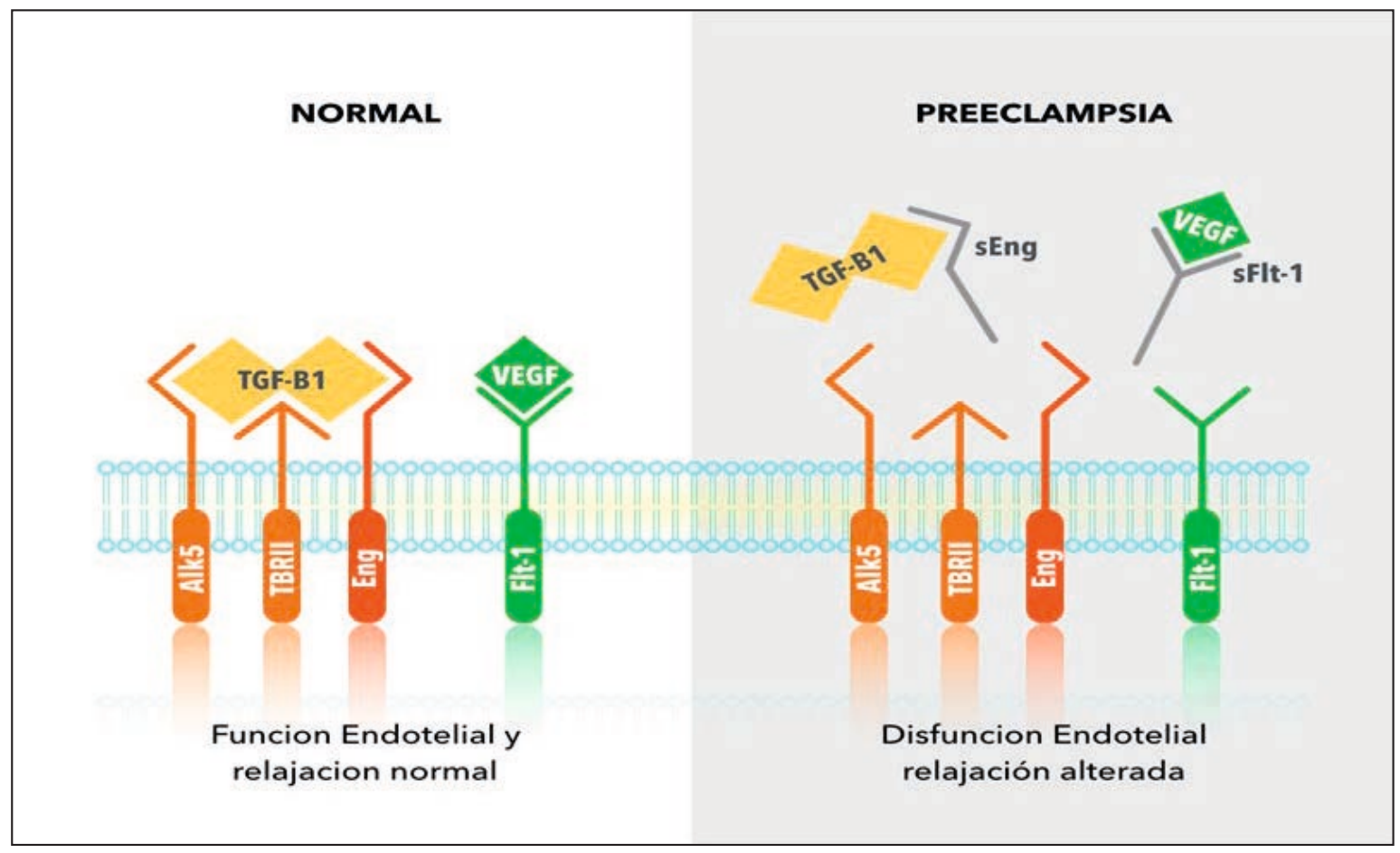

En la preeclampsia se ha encontrado un incremento precoz (antes de la etapa sintomática materna) de los factores antiangiogénicos principalmente sFlt-1 ${ }^{12,13,14}$. Estos altos niveles séricos de sFlt-1 bloquean la unión de VGEF y PGIF a sus receptores celulares produciendo y potenciando la disfunción del endotelio ${ }^{10}$. El aumento sérico materno de sFlt-1 tiene su origen en el trofoblasto extravelloso sometido a hipóxia crónica en las pacientes con preeclampsia, posiblemente incrementado por la acción del factor inducido por hipoxia-1 (HIF-1) que produce una regulación al alza del sFlt-1 en el trofoblasto ${ }^{9,15}$. Experimentos en ratas con administración de sFlt-1 exógeno desarrollan hipertensión, proteinuria y endoteliosis glomerular $^{16}$. Así también pacientes con cáncer sometidos a terapia anti-VEGF (bevacizumab) han presentado hipertensión, proteinuria y leucoencefalopatia posterior reversible ${ }^{17}$. Infusión de anticuerpos contra VEGF producen en ratones endoteliosis glomerular y proteinuria $^{18}$.

De la misma forma, existe aumento de los niveles séricos de sEng en los casos de gestantes con preeclampsia y estudios experimentales con administración de sFlt-
1 y sEng en ratas han producido: proteinuria, RCIU, hipertensión y aumento de la permeabilidad vascular ${ }^{19,20}$.

Los altos niveles de sFlt-1 en la preeclampsia tienen su origen al ser liberados desde la placenta y esto también puede explicar los bajos niveles séricos maternos de PIFG, al aumentar su unión no solo en el espacio intervelloso sino en el suero materno ${ }^{10}$. La producción de ON y la actividad de la sintetiza de ON se han encontrado disminuidas cuando existe elevación en suero materno de sFIt-1 y sEng en los casos con preeclampsia ${ }^{10,21}$.

Muchas citoquinas tienen influencia en la disfunción y activación endotelial tales como el TNF-alpha (factor de necrosis tumoral) y la IL-1, pero la complejidad de la inflamación y preeclampsia va mucho más allá de moléculas puntuales. Un ejemplo claro es la producción de auto-anticuerpos activantes de endotelina-1 producidos por linfocitos B en gestantes con preeclampsia, que aumentan la producción de sFlt-1 y sEng; lo que se ha relacionado a vasoconstricción y a daño cardiovascular a largo plazo ${ }^{10,22}$. (Figura 3). 
Figura 3. Factores angiogénicos y sus efectos en la disfunción endotelial que genera la sintomatología característica de la preeclampsia.

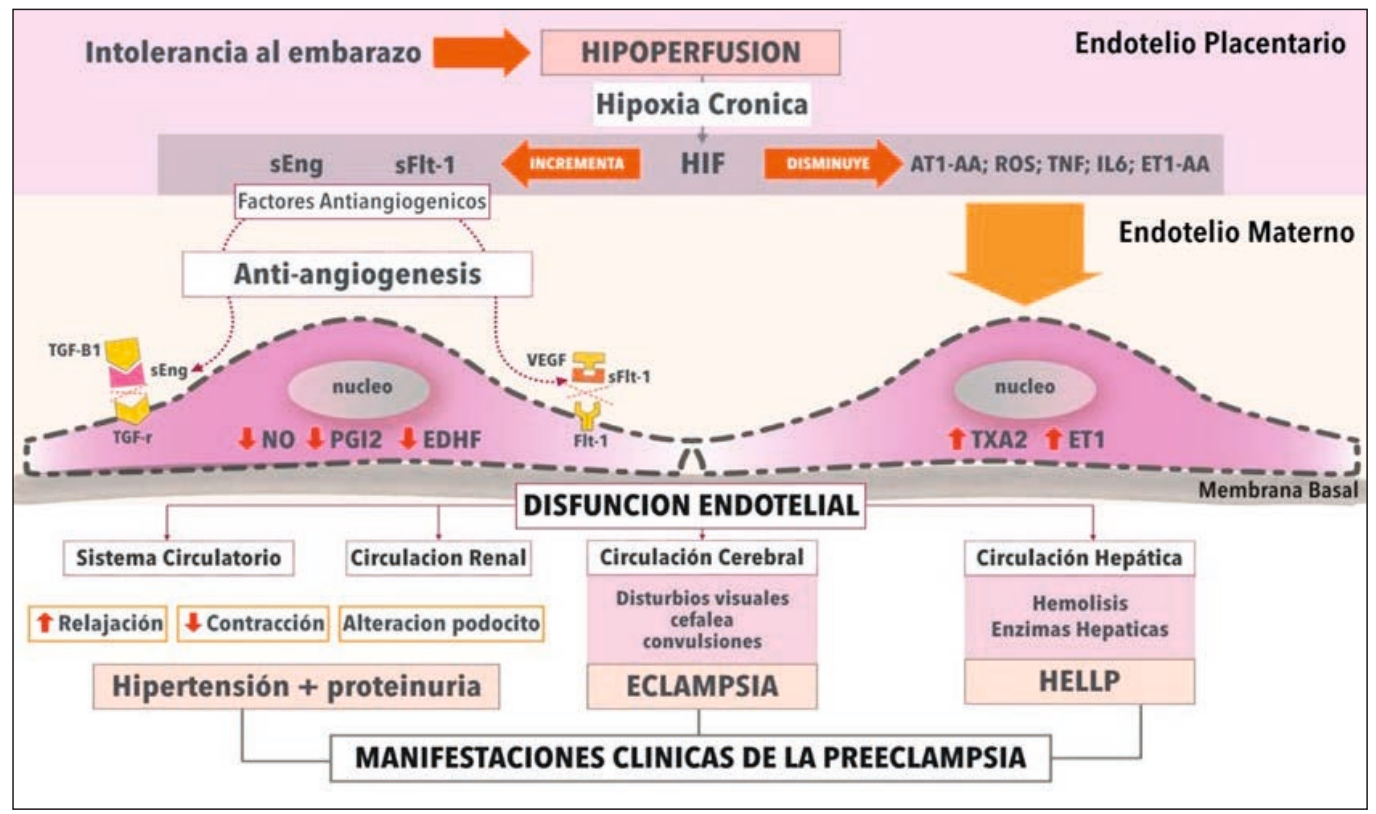

1. Ministerio de Salud. Resolución Ministerial 652-2016/MINSA (31.08.2016) Aprueba Norma Técnica N¹24-2016MINSA-V.01 Norma Técnica de Salud de Planificación Familiar. Perú, 2016.

Toda esta evidencia publicada en los últimos años hace suponer que existe un momento en la historia natural de la preeclampsia cuando se produce el desequilibrio angiogénico en la gestante (predominio de factores antiangiogénicos de origen placentario), lo que conlleva a producir disfunción endotelial. Posteriormente ya establecida la disfunción endotelial materna, dependiendo de los órganos afectados serán evidentes las manifestaciones clínicas que actualmente usamos como criterios diagnósticos (hipertensión arterial, proteinuria, etc). En estadios más avanzados de daño orgánico encontraremos los llamados criterios de severidad: daño renal (insuficiencia renal), afectación cerebral (cefalea, edema cerebral, convulsión, alteración del sensorio), daño pulmonar (edema agudo de pulmón), daño hepático (elevación de enzimas hepáticas que evidencian lisis celular).

Entonces, el evento fisiopatológico común en las gestaciones que desarrollan preeclampsia, independientemente del tiempo de inicio de la enfermedad, grado de severidad o velocidad de progresión, es el disbalance antiangiogénico y la disfunción endotelial como consecuencia de éste. Lo que queda por discutir a continuación es: ¿Cuál es el origen este disbalance en todos los fenotipos que encontramos en la preeclampsia?

\section{Estadio Asintomático: el origen del disbalance antiangiogénico}

La enfermedad placentaria de inicio temprano (arbitrariamente optaremos por el punto de corte antes de las 34 semanas) caracterizada habitualmente por preeclampsia severa y restricción de crecimiento fetal precoz; está asociada con frecuencia a isquemia e hipoperfusión placentaria, evidenciada en la histología placentaria por aterosis aguda, engrosamiento de la capa íntima, necrosis, ateroesclerosis, daño endotelial e infartos placentarios. Presumiblemente estos cambios se originan como consecuencia de la deficiente invasión perivascular y endovascular del trofoblasto en las arterias espirales uterinas, lo que ocasiona vasos de alta resistencia y con flujo turbulento de alta velocidad ${ }^{23}$.

El trofoblasto invasor normalmente expresa factores angiogenicos como el VEGF y el PIGF; sin embargo, los estudios de placentas de pacientes con preeclampsia han mostrado expresión anómala de estos factores angiogénicos, lo que podría explicar la deficiente capacidad invasora del trofoblasto en las paredes vasculares maternas (existe una delicada interacción entre factores inmunológicos, reconocimiento celular y señalización molecular que tiene influencia en la placentación, pero la revisión de ellos escapa a las objetivos de este articulo $)^{24}$. La consecuencia del fallo en la invasión trofoblástica será una placenta con hipoperfusión tisular crónica e hipoxia intermitente, generando estres oxidativo en el endotelio y trofoblasto de la vellosidad corial. La expresión y producción de sFlt-1 en estas circunstancias se incrementa mediado por el factor inducido por hipoxia - 1 (HIF-1) a nivel del trofoblasto ${ }^{10,15,23}$.

En la preeclampsia existe daño vascular placentario; sin embargo, el inicio del estadio sintomático materno (aparición de hipertensión arterial, proteinuria y daño endotelial) no solo depende de la presencia de daño placentario (hipoperfusión), si no de la severidad y de la magnitud del daño hipoxico (generando agravamiento del disbalance antiangiogénico conforme progresa la gestación $)^{25,26}$. Un ejemplo llamativo y extremo es el 
embarazo molar, reconocido como una de las "excepciones" para desarrollar preeclampsia antes de las 20 semanas, esto se explica al considerar a la placenta con degeneración hidrópica como el extremo máximo de la insuficiencia placentaria (avascular), desarrollando preeclampsia en su presentación más precoz ${ }^{26}$

Podemos entonces establecer una cadena de eventos fisiopatológicos coherentes: la deficiente invasión trofoblastica produce a lo largo de la gestación hipoperfusion tisular e hipoxia placentaria, esto genera el posterior incremento de la expresión y producción de factores antiangiogénicos (entre otros factores) en el espacio intervelloso, llevándonos a la circulación materna y generando disfunción endotelial sistémica ${ }^{27}$.

Sin embargo, este modelo solo explicaría a la preeclampsia de inicio temprano donde la prevalencia de hipoxia placentaria es mucho más alta. En la preeclampsia de inicio tardío (mayor de 34 semanas) a diferencia del fenotipo de inicio temprano la hipoperfusión placentaria parece ser menos relevante ${ }^{28}$; confirmando este hecho, vemos que las gestaciones con preeclampsia antes de las 27 semanas demuestran daño histológico placentario en un $75-100 \%$, en comparación al $13 \%$ de las gestaciones que desarrollaron preeclampsia a las 41 semanas $^{29}$.

Para poder entender porque en una gestación con aparente placentación normal y crecimiento fetal adecuado experimenta desequilibrio angiogénico debemos considerar a la preeclampsia como un síndrome feto-materno "adaptativo". Desde el lado fetal podemos especular que el objetivo sería incrementar de manera "compensatoria" la presión arterial sistémica materna y así mejorar el flujo útero-placentario para cumplir las demandas fetales. Page propone la hipótesis: "Si la placenta no pudiera obtener una circulación materna suficiente para sus demandas, podría ser capaz de aumentar esta oferta elevando la presión arterial sistémica", de esta forma garantizaría una adecuada nutrición fetal ${ }^{30}$.

El feto ante una deficiente nutrición puede "comunicarse" con su placenta mediante señalización endotelial (al existir continuidad entre endotelio vascular fetal, cordón umbilical y vellosidades coriales) produciendo el incremento de los factores antiangiogenicos si fuera requerido (perdida del equilibrio en el feedback feto-materno $)^{11}$. En este sentido, se ha propuesto a la adenosina endotelial fetal como una posible señal fetal, dado que en estudios experimentales demostró incrementar la expresión placentaria de sFlt-1 en condiciones de normoxia e hipoxia ${ }^{31,32}$. Desde el lado materno existe una fuerte asociación entre preeclampsia de inicio tardío y condiciones maternas con disfunción endotelial de otro origen (hipertensión crónica, diabetes, obesidad, etc); en este contexto un leve disbalance antiangiogénicos produciría agravamiento de la disfunción endotelial y en consecuencia el síndrome materno se haría evidente ${ }^{33,34}$. Tal es así que la cuantía de la elevación de sFlt-1 es mucho mayor en los casos de inicio temprano en comparación a los casos de inicio tardío ${ }^{35}$; por ello al ser más leve el desequilibrio y la disfunción endotelial producida, la sintomatología materna también sería menos severa en comparación a los casos de inicio temprano, hecho que es apoyado por los datos epidemiológicos del comportamiento clínico de la preeclampsia de inicio tardío ${ }^{26,36}$.

En conclusión, los factores angiogénicos son moléculas vitales dentro del delicado equilibrio vascular adaptativo que representa una gestación normal. Los orígenes de la preeclampsia son demasiado complejos para poder ser explicados por una única molécula (o familia de moléculas), por ello aun no podemos esclarecer completamente la cadena de eventos fisiopatológicos que generan en la madre la disfunción endotelial. El desequilibrio angiogénico es actualmente un prometedor camino que podría integrar las múltiples teorías y acércanos a un futuro tratamiento de la preeclampsia.

\section{Financiamento: Autofinanciado}

Conflicto de interés: Los autores declaran no tener ningún conflicto de intereses.

\section{REFERENCIAS BIBLIOGRÁFICAS}

1. Sibley CP. Treating the dysfunctional placenta. J Endocrinol 2017;234(2):R81-R97. doi: 10.1530/JOE-17-0185 doi: 10.1530/JOE-17-0185.

2. Bell MJ. A historical overview of preeclampsia-eclampsia. J Obstet Gynecol Neonatal Nurs. 2010;39(5):510-8. doi: 10.1111/j.1552-6909.2010.01172.x.

3. Lacunza RO, Pacheco-Romero J. Implicancias neurológicas de la preeclampsia, más que solo eclampsia. Rev Per Ginecol Obstet. 2015;61(4):407-416.

4. Lacunza RO, Santis F. Sulfato de magnesio y el cerebro en la preeclampsia. Rev Per Ginecol Obstet. 2017;63(2):235240.

5. Possomato-Vieira JS, Khalil RA. Mechanisms of Endothelial Dysfunction in Hypertensive Pregnancy and Preeclampsia. Adv Pharmacol 2016;77:361-431. doi: 10.1016/ bs.apha.2016.04.008

6. Shah DA, Khalil RA. Bioactive factors in uteroplacental and systemic circulation link placental ischemia to generalized vascular dysfunction in hypertensive pregnancy and preeclampsia. Biochem Pharmacol 2015.15;95(4):211-26. doi: 10.1016/j.bcp.2015.04.012.

7. Boeldt DS, Bird IM. Vascular Adaptation in Pregnancy and Endothelial Dysfunction in Preeclampsia. J Endocrinol 2017;232(1):R27-R44 doi: 10.1530/joe-16-0340

8. Page EW. The effect of eclamptic blood upon the urinary output and blood pressure of human recipients. J Clin Invest 1938;17(3):207-18. Doi: 10.1172/JCl100945

9. Nagamatsu T, Fujii T, Kusumi M, Zou L, Yamashita T, Osuga Y, et al. Cytotrophoblasts up-regulate soluble fms-Like tyrosine kinase-1 expression under reduced oxygen: an implication for the placental vascular development and the pathophysiology of preeclampsia. Endocrinology 2004;145(11):483845. Doi: 10.1210/en.2004-0533

10. Brennan LJ, Morton JS, Davidge ST. Vascular Dysfunction in Preeclampsia. Microcirculation 2014;21(1):4-14. doi: 10.1111/micc.12079.

11. Fan X, Rai A, Kambham N, Sung JF, Singh N, Petitt M, et al. Endometrial VEGF induces placental SFLT1 and leads to pregnancy complications. J Clin Invest 2014;124(11):494152. doi: $10.1172 / \mathrm{JCl} 76864$.

12. Bian Z, Shixia C, Duan T. First-trimester maternal serum levels of SFLT1, PGF and ADMA predict preeclampsia. PLoS One 2015.23;10(4):e0124684. doi: 10.1371/journal. pone.0124684

13. March MI, Geahchan C, Wenger J, Raghuraman N, Berg A, Haddow $\mathrm{H}$, et al. Circulating angiogenic factors and the risk of adverse outcomes among Haitian women with preeclampsia. PLoS One 2015.12;10(5):e0126815. doi: 10.1371/journal.pone. 0126815 .

14. McKeeman GC, Ardill JE, Caldwell CM, et al. Soluble vascular endotelial growth factor receptor-1 (sFIt-1) is increased 
throughout gestation in patients who have preeclampsia develop. Am J Obstet Gynecol. 2004;191:1240-46 doi: 10.1016/j.ajog.2004.03.004

15. Anton L, Olarerin-George AO, Hogenesch JB, Elovitz MA. Placental expression of $\mathrm{miR}-517 \mathrm{a} / \mathrm{b}$ and $\mathrm{miR}-517 \mathrm{c}$ contributes to trophoblast dysfunction and preeclampsia. PLoS One 2015.23;10(3):e0122707. doi: 10.1371/journal. pone. 0122707

16. Holwerda KM, Burke SD, Faas MM, Zsengeller Z, Stillman IE, Kang PM, et al. Hydrogen sulfide attenuates sFlt1-induced hypertension and renal damage by upregulating vascular endothelial growth factor. J Am Soc Nephrol 2014;25(4):71725. doi: 10.1681/ASN.2013030291

17. Zhu X, Wu S, Dahut WL, Parikh CR. Risks of proteinuria and hypertension with bevacizumab, an antibody against vascular endothelial growth factor: Systematic review and meta-analysis. Am J Kidney Dis 2007:49(2):186-93. Doi: 10.1053/j.ajkd.2006.11.039

18. Eremina V, Jefferson JA, Kowalewska J, Hochster H, Haas $\mathrm{M}$, Weisstuch J, et al. VEGF inhibition and renal thrombotic microangiopathy. N Engl J Med 2008.13;358(11):1129-36. doi: 10.1056/NEJMoa0707330.

19. Ramma W, Buhimschi IA, Zhao G, Dulay AT, Nayeri UA Buhimschi CS, et al. The elevation in circulating anti-angiogenic factors is independent of markers of neutrophil activation in preeclampsia. Angiogenesis 2012;15(3):333-40. doi: 10.1007/s10456-012-9261-5

20. Venkatesha S, Toporsian M, Lam C, Hanai J, Mammoto T, Kim YM, et al. Soluble endoglin contributes to the pathogenesis of preeclampsia. Nat Med 2006;12(6):642-9 doi: 10.1016/s1090-798x(08)70058-4

21. Sandrim VC, Palei AC, Metzger IF, Gomes VA, Cavalli RC Tanus-Santos JE. Nitric Oxide Formation Is Inversely Related to Serum Levels of Antiangiogenic Factors Soluble Fms-Like Tyrosine Kinase-1 and Soluble Endogline in Preeclampsia. Hypertension 2008;52(2):402-7. doi: 10.1161/ HYPERTENSIONAHA.108.115006.

22. Hubel CA, Wallukat G, Wolf M, Herse F, Rajakumar A, Roberts JM, et al. Agonistic angiotensin II type 1 receptor autoantibodies in postpartum women with a history of preeclampsia. Hypertension 2007;49: 612-617 doi: 10.1161/01. hyp.0000256565.20983.d4

23. Burton GJ, Woods AW, Jauniaux E, Kingdom JC. Rheological and physiological consequences of conversion of the maternal spiral arteries for uteroplacental blood flow during human pregnancy. Placenta. 2009;30(6):473-82. doi: 10.1016/j.placenta.2009.02.009

24. Mateus J. Significancia del desbalance de los factores angiogénicos en preeclampsia. Rev Per Ginecol Obstet 2014;60(4):333-343

25. Espinoza J. Angiogenic imbalances in the pathogenesis of pregnancy complications. Fetal Matern Med Rev 2014; 25(1):42-58 doi: 10.1017/s0965539514000096
26. Espinoza J, Uckele JE, Starr RA, et al. Angiogenic imbalances: the obstetric perspective. Am J Obstet Gynecol 2010; 203:17.e1-8. Doi: 10.1016/j.ajog.2009.10.891

27. Holme AM, Roland MC, Henriksen T, Michelsen TM. In vivo uteroplacental release of placental growth factor and soluble Fms-like tyrosine kinase-1 in normal and preeclamptic pregnancies. Am J Obstet Gynecol 2016;215(6):782.e1-782.e9. doi: 10.1016/j.ajog.2016.07.056

28. Lacunza RO, Pacheco-Romero J. Preeclampsia de inicio temprano y tardío: una antigua enfermedad, nuevas ideas. Rev Per Ginecol Obstet. 2014;60(4): 351-362

29. Ogge G, Chaiworapongsa T, Romero R, Hussein Y, Kusanovic J P, Yeo L, Kim C J, Hassan S. Placental lesions associated with maternal underperfusion are more frequent in early-onset than in late-onset preeclampsia. J Perinat Med. 2011; 39(6):641-652 doi: /10.1097/ogx.0b013e31824b7043

30. Page EW. The relation between hydatid moles, relative ischemia of the gravid uterus, and the placental origin of eclampsia. Am J Obstet Gynecol 1939; 37:291-293.

31. Espinoza J, Espinoza AF. Pre-eclampsia: a maternal manifestation of a fetal adaptive response? Ultrasound Obstet Gynecol. 2011;38(4):367-70. doi: 10.1002/uog.10067

32. George EM, Cockrell K, Adair TH, Granger JP. Regulation of sFlt-1 and VEGF secretion by adenosine under hypoxic conditions in rat placental villous explants. Am J Physiol Regul Integr Comp Physiol 2010; 299: R1629-R1633. Doi: 10.1152/ajpregu.00330.2010

33. Ferrazzi E, Stampalija T, Aupont JE. The evidence for late onset preeclampsia as a maternogenic disease of pregnancy. Fetal Matern Med Rev 2013; 24 (1): 18-31 doi: 10.1017/ s0965539513000028

34. Spradley FT, Palei AC, Granger JP. Immune mechanisms linking obesity and preeclampsia. Biomolecules. 2015.12;5(4):3142-76. doi: 10.3390/biom5043142.

35. Wikstrom AK, Larsson A, Eriksson UJ, et al. Placental growth factor and soluble fms-like tyrosine kinase-1 in early-onset and late-onset preeclampsia. Obstet Gynecol. 2007;109(6):1368-74. Doi: 10.1097/01. aoa.0000308300.91284.36

36. Phillips J, Janowiak M, Badger G, Bernstein I. Evidence for distinct preterm and term phenotypes of preeclampsia. J Matern Fetal Neonatal Med. 2010;23(7):622-626 doi: $10.1080 / 14767050903258746$

\section{Correspondencia:}

Rommel Omar Lacunza Paredes

Dirección: Av. Guardia Chalaca 2176, Bellavista. Callao

Celular: 985436784

Correo electrónico: drrlacunza@hotmail.com 